\title{
Evaluation of the Performance of Spontaneously Deployed, Independent Networks
}

\author{
Ivan Gudymenko, Waltenegus Dargie and Alexander Schill \\ Chair for Computer Networks \\ Faculty of Computer Science \\ Technical University of Dresden \\ 01062 Dresden, Germany \\ Email: (ivan.gudymenko@gmail.com, waltenegus.dargie, alexander.schill)@tu-dresden.de
}

\begin{abstract}
The growing demand for a wireless link is taking the deployment of wireless local area networks away from the notion of carefully planned and carefully managed setting, into a randomly deployed and independently managed (if at all) network setting. This results in highly contentious networks. In fact research shows that in most metropolitan cities, the size of contentious access points that are closely located with each other are overwhelmingly high. Subsequently, the performance of these networks is highly fluctuating and unpredictable. This paper aims to show how spontaneously deployed networks perform in the presence of fluctuating node densities. We perform an exhaustive simulation for different applications, including, VoIP, HTTP, and FTP. We focus on non-commercial deployments (private use at home or in an apartment).
\end{abstract}

\section{INTRODUCTION}

Over the past few years, wireless networking has become an inseparable part of users' everyday life. In the past, wireless local area networks have been deployed mainly by enterprises and big companies, with careful planning and radio resource management strategies. Presently, the deployment of unplanned and unmanaged wireless local area networks is commonplace. Most of these networks are non-commercial and share overlapping channels. These types of wireless deployments usually have no special managing and planning tools for cost and complexity reasons. They inevitably cause mutual interference which in turn is the cause of overall network performance degradation.

Several strategies have been proposed to improve service qualities, including increasing the transmission power as the signal to noise ration declines below a certain threshold; dynamic channel allocation, and dynamic rate adaptation. Depending on the purpose and the densities of the spontaneously networks, these strategies may or may not be effective. Even when they are effective, it is usually at the expense of some other performance or efficiency metric. For example, increasing the transmission power of an access point when the level of interference becomes significantly high may improve the signal to noise ratio; however, the same decision maybe made by contending access points, as a result, there will be no net gain in the SNR. Moreover, even if there is a gain with this strategy, it is achieved by inefficiently utilizing the available energy. In general, proposed strategies have some upper bound, beyond which they may not be able to provide any appreciable improvement in performance.

The aim of this paper is to investigate the performance of spontaneously deployed networks when different types of applications (HTTP, FTP, and VoIP) run on terminal devices access the network. Moreover, we consider different strategies to observe the net gain in performance or in energy efficiency. The simulation results will be useful to recognize the types of applications that are significantly affected by the networks' setup. Additionally, the simulation will be used to demonstrate the scope and usefulness of the strategies that are proposed to meaningfully utilize a shared channel. This investigation will be a basis for applying self-managing strategies [8], [14] to improve performance and the stability of spontaneously deployed networks.

The rest of the paper is structured as follows. Section II presents related work; section III, presents the model of the networks that are spontaneously deployed as well as the basic assumptions for setting up the simulation environment. In section IV, the simulation environment and the simulation results are presented in detail. Finally, in section V, a concluding remark will be given.

\section{RELATED WORK}

According to ABI research [13], the global growth of Wi-Fi hotspots in 2008 is estimated to be $40 \%$ higher than in 2007. The greatest growth and the largest number of hotspots are observed in Europe. Moreover, it is claimed that the dominant form of networking connectivity for consumer electronics is likely to be Wi-Fi as the most widely used type of consumerinstallable and retail-based networking.

In [1], an empirical study (based on several metropolitan cities in the US) of unplanned and unmanaged networks is discussed in detail. The paper shows that most of the access points are deployed with default setting. It also observes that even though there automated network management software for (re)configuring APs, most of these products are tailor made for specific hardware.

The problem of interference in 802.11 Networks is discussed in [12], [9]. Accordingly, several strategies are suggested to mitigate the problem. The first strategy is capacity planning using relevant software packages that allow modeling the environment, identifying the ceiling height, number of 
floors, material types of the walls, doors etc., and defining coverage zones with specific bandwidth. The second strategy is to use multiple service set identifiers (SSIDs) and thus assigning the guest traffic to the SSID with limited bandwidth and low priority Quality of Service. Both strategies are not feasible to implement for non-commercial, spontaneously deployed networks. There are two reasons for this: (1) there are no special management tools available; and (2) all the networks are independent and may not be willing to cooperate.

In [9], the benefits of channel allocation in 802.11 networks capacity enhancement and channel failure recovery (due to frequency diversity) are discussed. However, all the simulations are performed for ad-hoc networks. In contrast, the focuses of this paper are AP-based, spontaneously deployed networks where no additional hops are possible or desirable.

In [10] the performance of 802.11 networks under high density condition is investigated. It is shown that in such condition, jointly tuning power level and the carrier sensing parameter of the MAC layer of the nodes in conjunction with cross-layer approach is preferable.

\section{Network Parameters AND Models}

The performance of a wireless network depends on various factors and network parameters [16]. At the physical layer, interference is the main limiting factor. To deal with interference, the strategies that are often employed are dynamic channel allocations, rate adaptation and dynamic control of transmission power.

At the link layer, contention, and thereby, packet collision and packet retransmission, are the main limiting factors [6]. To minimize the effect of collision, there are collision avoidance techniques in place. However, most of these techniques employ control packet overhead which result in potential delay and, in case of infrequent contention, a reduction in overall throughput and an inefficient use of energy. Subsequently, the decision to use control packet overheads depends on node density, the type of applications that access the network, and the frequency of communication. For example, empirical study shows that most IEEE 802.11 based wireless local area networks avoid collision avoidance techniques (they disable the sending of RTS/CTS control packet overheads), preferring retransmission if collision occurs and a packet is lost [1].

We aim to observe the performance of spontaneously deployed networks by investigating the impact of several adaptation strategies at the application, link and physical layers. At the application layer we model different types of applications, both for a constant bit rate and variable bit rate models: ftp (represents an "aggressive" traffic and attempts to occupy the entire available bandwidth for transmission), VoIP (traffic with special requirements of connection stability parameters) and HTTP (representing a bursty traffic of Internet browsing).

At the link layer, we consider a network setup both with and without control packet overheads. At the link layer, we consider three channel conditions: (1) where the various access points of independent networks are able to allocate channels that are not occupied; (2) where there is an arbitrary allocation

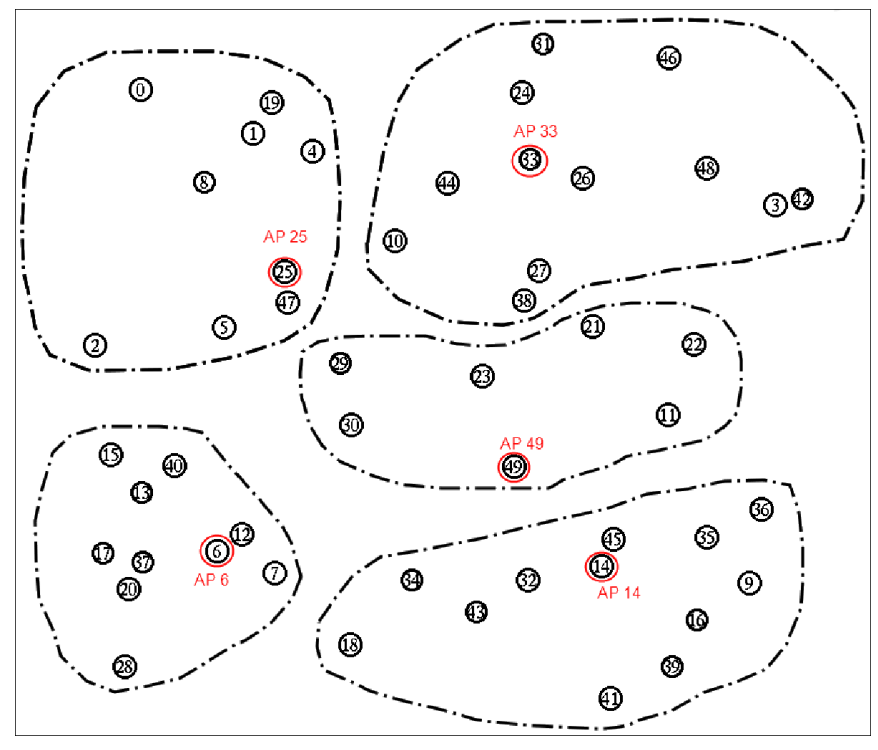

Fig. 1. Network topology.

of overlapping channels between independent access point; and (3) where there is a hybrid channel allocation, i.e., some access points occupy non-overlapping channels and others share channels.

The model of a network establishes the basic assumptions concerning the distribution of nodes, the node density and the different types of constraints at the application, the link and the physical layers. The entire area that is occupied by the spontaneously deployed networks can be modeled as a 2-dimensional Poisson distributed network (figure 1) [3], [11], and [5]. Therefore, as shown in figure 1, $N$ nodes are distributed randomly on a rectangular area $A$ of size $A=a \times b$, where $a \leq b$. The average node density is denoted by $\lambda$. The probability of finding $k$ nodes in $A$ is given by:

$$
P(k \text { nodes } \in A)=e^{\lambda A} \frac{(\lambda A)^{k}}{k !}
$$

The connectivity figure speaks about the existence of a communication link between a child node anywhere in the specified field and an arbitrary access point, such that there is a direct link between the child node and the access point. For homogeneous nodes, the relationship between connectivity probability, transmission range and node density is estimated by [2]:

$$
r_{0} \cong \sqrt{\frac{\ln \left(1-P(\text { conn })^{\frac{1}{n}}\right)}{-\lambda \pi}}
$$

where Pconn is the probability that the network is connected; $\lambda$ is the density of the network, $n / A ; r_{o}$ is the threshold transmission rage; and $n>>1$ is the number of deployed nodes. Each node has the same radio transmission range $R$, and a node can directly communicate with an access point if their Euclidean distance is less than the transmission range, 


\begin{tabular}{|l|l|l|l|l|}
\hline $\begin{array}{l}\text { Simulation } \\
\text { setting }\end{array}$ & 1 & 2 & 3 & 4 \\
\hline $\begin{array}{l}\text { Channels } \\
\text { assigned }\end{array}$ & $1,6,11$ & $1,6,11$ & $1,3,6,8,11$ & 1 \\
\hline RTS/CTS (on/off) & OFF & ON & OFF & OFF \\
\hline
\end{tabular}

TABLE I

Simulation SETTING

\begin{tabular}{|l|l|l|}
\hline FTP & VoIP & HTTP \\
\hline $60 \%$ & $20 \%$ & $20 \%$ \\
\hline
\end{tabular}

TABLE II

NODE DENSITY FOR DIFFERENT APPLICATIONS

i.e., $d \leq R$. For simplification, fading and path efficiency are not taken into account.

\section{SIMULATIONS}

\section{A. Setting description}

We used the Network Simulator 2 (NS-2) [7] to perform the simulation. There are several implementations of 802.11 standard in NS-2. Ours is based on the enhanced MAC module, Mac/80211ext [4], which enables a more realistic modeling environment.

The setting of the simulations is described as follows: 50 mobile terminals are randomly deployed in an area of $100 \mathrm{x}$ $100 \mathrm{~m}^{2} .{ }^{1}$ These 50 mobile terminals (MT) form 5 independent AP-based networks. Five of these (terminal 25, 6, 33, 49 and 14) play the role of an access point, while the rest connect to them to form spontaneous AP-based wireless networks. This is depicted in figure 1.

We consider various network setups to study the throughput of collective and individual networks: the types of traffic used by the mobile terminals (FTP, VoIP and HTTP); the channel allocation mechanism (single channel, multiple channels; overlapping channels, non-overlapping channels); and the presence or absence of control packet overheads (i.e., the RTS/CTS mechanism is turned on or off). Tables I and II summarize the simulation settings.

According to table $\mathrm{I}$, in settings 1 and 2, the networks occupy non-overlapping channels; in setting 3 , there is a partal occupation of non-overlapping channels; and in setting 4, all the access points occupy a single channel, to demonstrate the worst case scenario. In order to simulate FTP traffic, the FTP over TCP model is employed. To provide simulations of VoIP traffic, the constant bit rate (CBR) traffic implementation over UDP is chosen. Subsequently, the packet size is $=80$ and the transmission rate is $=64 k b p s$. The HTTP traffic is simulated using the exponential traffic model that is implemented in NS2. The simulation time is set to 50 seconds.

\footnotetext{
${ }^{1}$ Our network model is assumed to be a 2-D. This however, should not be considered as an over simplification. Given the relatively short communication distance of nodes with their access points, the actual 3-D setting can be modelled by a $2 \mathrm{D}$ setting without any loss of vital information.
}

\section{B. Performance}

In general, two important observations have been made in the simulation. Firstly, the simulations confirms that the lack of a systematic channel allocation mechanism is a reason for the dramatic degrading of the performance of spontaneously deployed networks. Secondly, collision avoidance mechanism, which are effective in dealing with hidden terminals in IEEE 802.11 networks, contribute only slightly. This is because, nodes are distributed in very close proximity that the packet collision due to hidden terminals in less significant.

In the following subsection, a more detailed description of four different settings will be given.

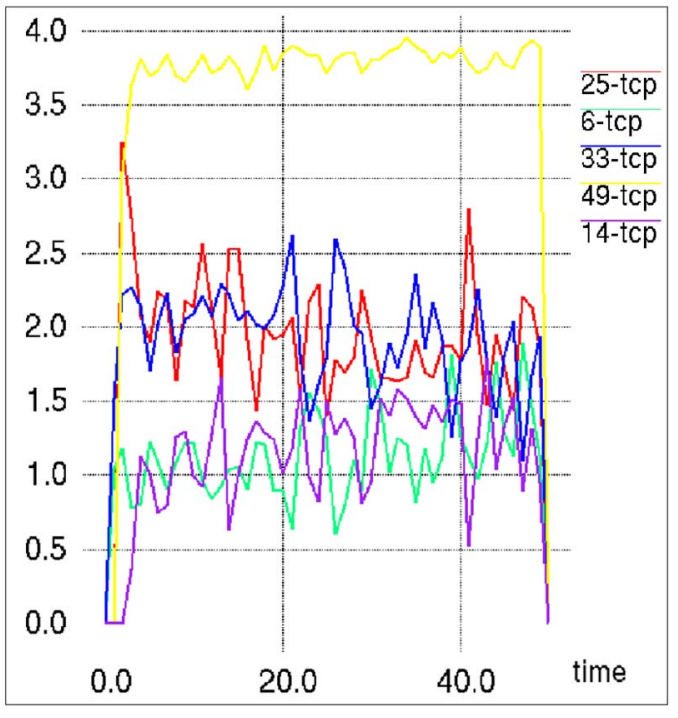

Fig. 2. FTP Traffic

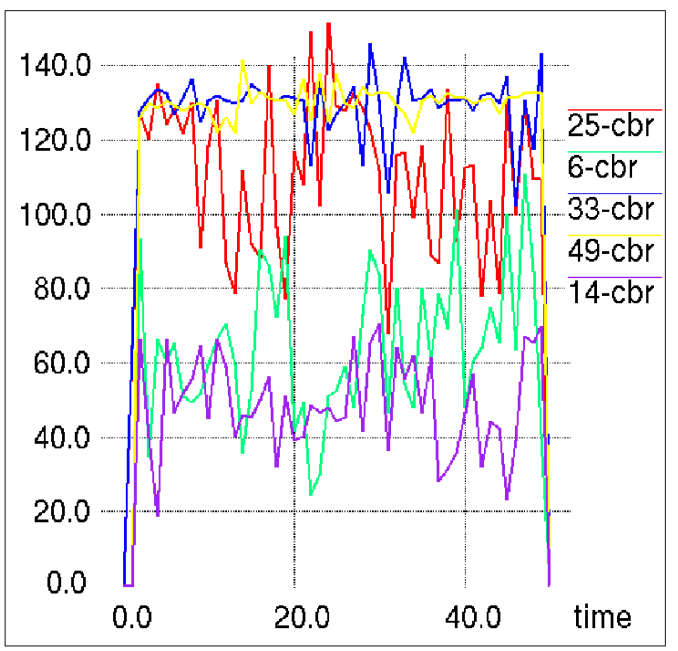

Fig. 3. VoIP traffic (CBR packets received by each AP)

1) Setting 1. (channels 1, 6, 11; RTS/CTS OFF): In this setting the networks are operating on channels 1,6 , and 11 . Namely, channels 1 and 11 are shared by two networks which 
are not direct neighbors and channel 6 is used by the fifth network, which is in the center of the simulation topology (see fig. 1). The contention avoidance mechanism is turned off. The node density for different applications is as described in table II.

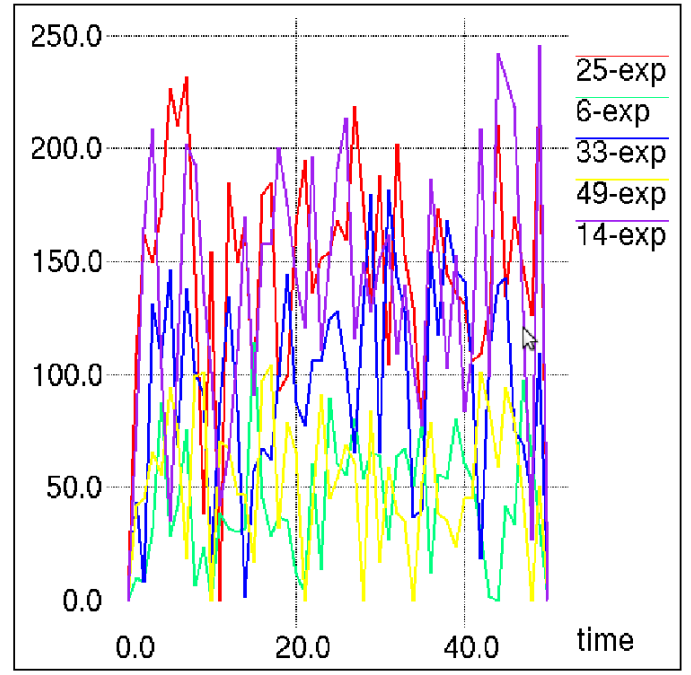

Fig. 4. HTTP traffic emulation.

In fig. 2, 3, and 4, the overall network throughput (the number of packets generated by the three applications - ftp, VoIP, HTTP - and received by the AP is depicted. The performance of the network with the AP 49 is significantly higher (up two times better) and more stable compared with the other networks. This is due to the fact that collisions and inter network interference (analogous to inter-cell interference in GSM) leads to the degradation of the overall network performance and throughput. In fig. 2, the FTP traffic is presented. On the one hand, the FTP attempts to capture the available bandwidth, and therefore, the collision rate and traffic fluctuation are relatively high; on the other hand, FTP is not a time-critical application, because the requirements for average delay and jitter (traffic stability) are not as strict as that of VoIP. In fig. 3, which represents VoIP traffic, a similar characteristic can be observed: the performance of the network which does not share the channel with any other network is relatively high and stable. But the other networks' performance is not at all good. The fluctuation of VoIP traffic is substantial, which implies that the link quality is not at all desirable (high jitter and long delays). The fluctuation of the HTTP traffic 4 is the worst. We modeled this traffic as a bursty traffic on purpose.

2) Setting 2. (channels 1, 6, 11; RTS/CTS ON): This setting is similar to the previous one except for the fact that the RTS/CTS mechanism is turned on. The figures 5, 6, and 7 show the impact of collision avoidance mechanism on the overall networks performance. The impact does not appear to be profound. This fact can be explained as follows. The RTS/CTS control overhead in IEEE 802.11 wireless networks is introduced basically to reduce frame collisions that are caused by hidden terminals. However, in our scenario node density is relatively high, which means that the distance between neighboring nodes is relatively small. Subsequently, collision due to hidden terminals is relatively small.

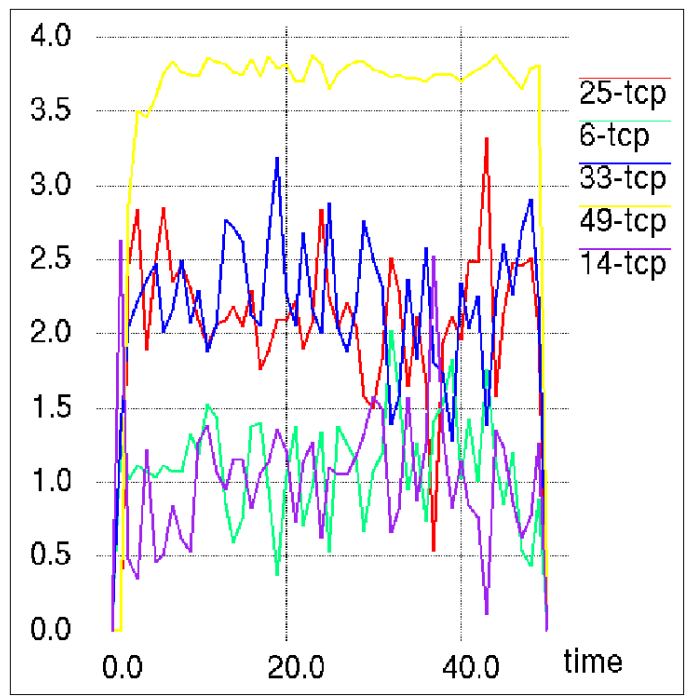

Fig. 5. FTP traffic (tcp packets received by each AP).

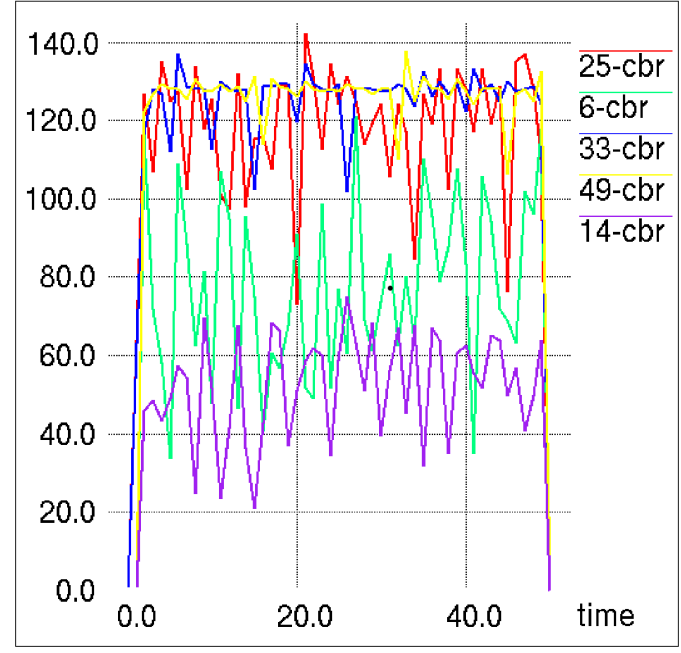

Fig. 6. VoIP traffic (cbr packets received by each AP)

3) Setting 3. (channels 1, 3, 6, 8, 11; RTS/CTS OFF): In this setting the networks are operating on different channels (non-overlapping channels). The collision avoidance mechanism is turned off. The node density for different applications is as described in table II.

The substantial increase in the performance for the three applications can be observed in fig. 8, 9 and 10). Fig. 8 shows the fluctuation of the overall performance of the FTP application. Obviously, the result is much better than the performance of the other networks. With non-overlapping channel, the medium is so efficiently utilized such that the overall performance of all the networks is almost identically good. The collision rate is relatively low due to separate 


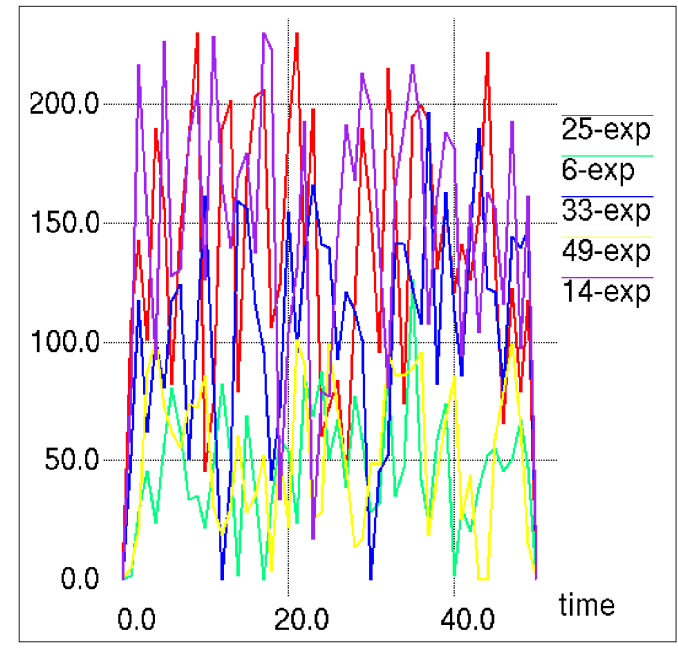

Fig. 7. HTTP traffic emulation.

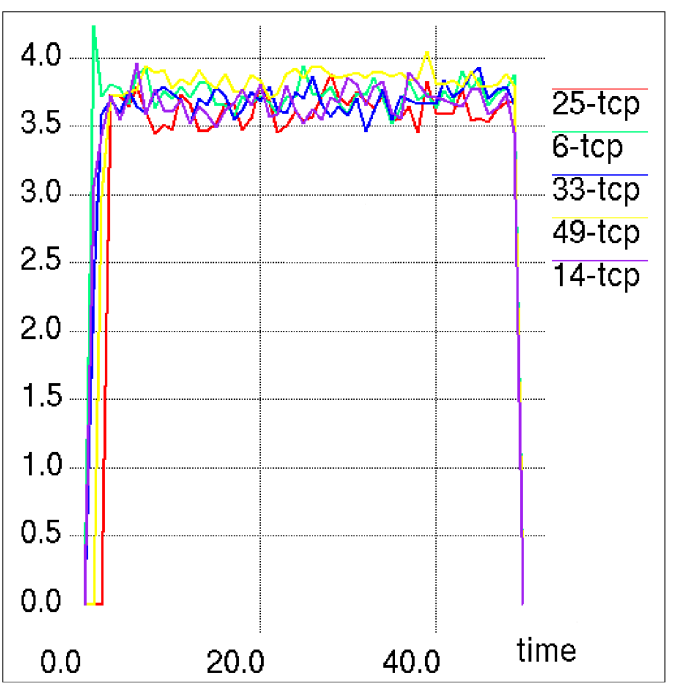

Fig. 8. FTP traffic (tcp packets received by each AP).

channels utilization. Fig. 9 represents the characteristic of the networks for the VoIP application. The stability parameters (jitter and fluctuation) appear to be far more better than the other settings. This demonstrates the significance of using nonoverlapping channels in spontaneously deployed networks.

4) Setting 4. (single channel operation; RTS/CTS OFF): In this setting all the networks share a single channel, to demonstrate the worst case scenario. The collision avoidance mechanism is also turned off. The overall network performance degrades dramatically. The performance of the FTP is particularly poor. The FTP traffic attempts to utilize the maximum available bandwidth, as a result it resembles the HTTP traffic in previous settings: burtsy and highly unstable. This is due to an extremely high collision rate. The average jitter and delay parameters of the VoIP render spontaneously deployed wireless networks unsuitable for VoIP applications. The HTTP traffic can still be supported by such a setting.

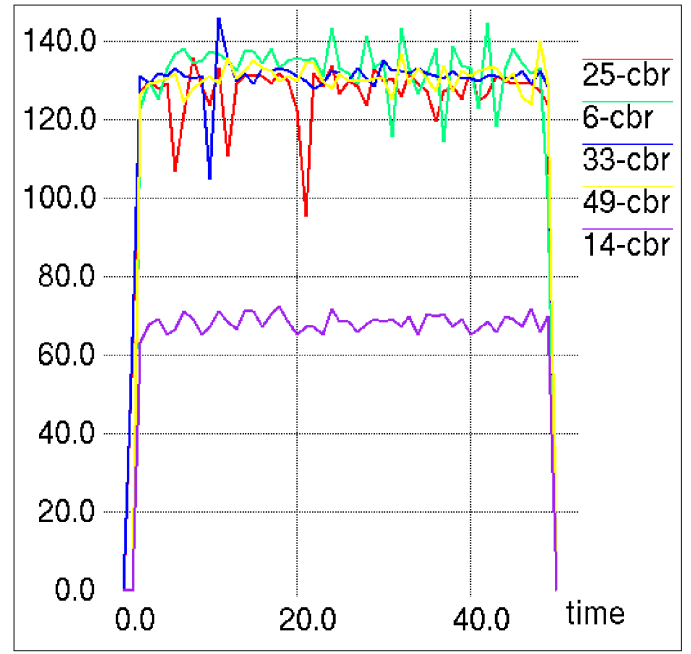

Fig. 9. VoIP traffic (cbr packets received by each AP).

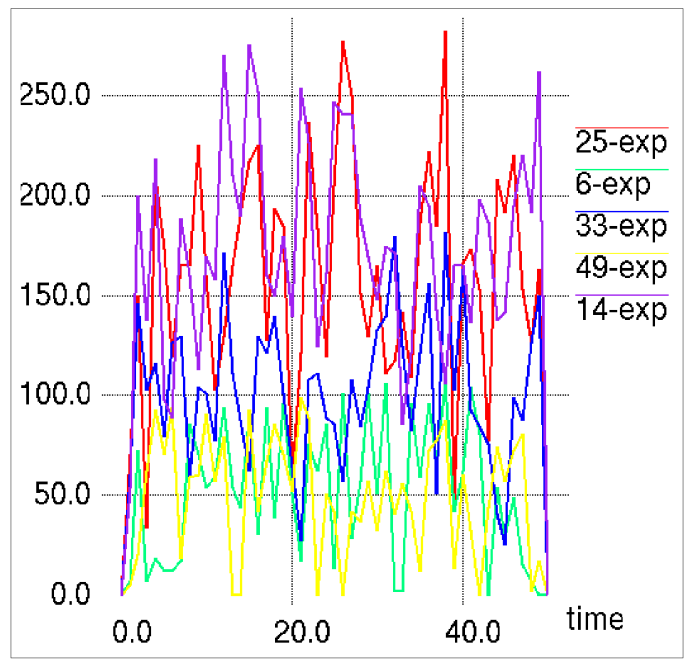

Fig. 10. HTTP traffic emulation.

\section{CONLUSION}

We investigated the performance of spontaneously deployed networks by considering various deployment settings. The simulation was carried out in NS-2 environment and the following observations were made. Of all the parameters, dynamic channel allocation improves or affects the performance of spontaneously deployed networks. This shows that inter-channel interference is more significant than the intrachannel problem of collision. This statement is confirmed by observing the performance of the collision avoidance mechanisms (RTC/CTS) in different deployment settings. For this reason, it is highly advisable that the channel allocation mechanism is applied in the first place when collision and interference mitigation is required. In most cases, switching the control packet overheads on or off produces little difference on the networks' performance. Subsequently, implementing simple dynamic channel allocation mechanisms is more useful than implementing collision avoidance mechanism. In fact, 


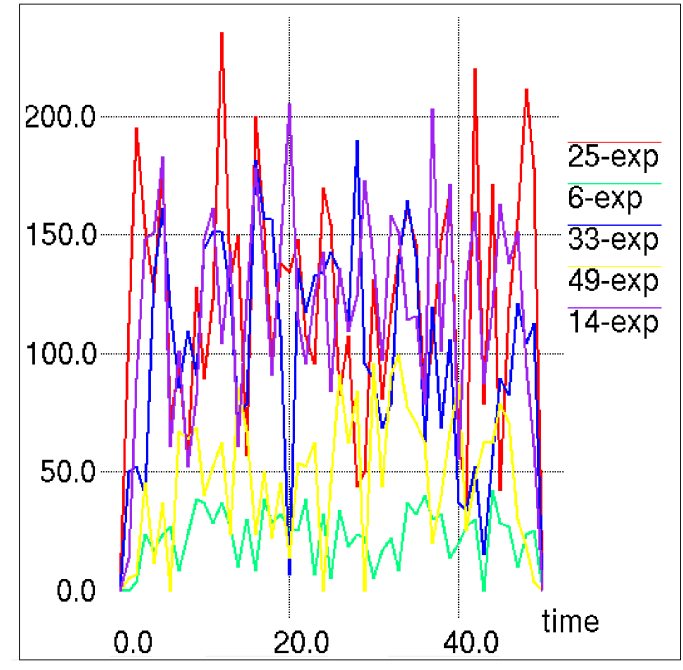

Fig. 13. HTTP traffic emulation.

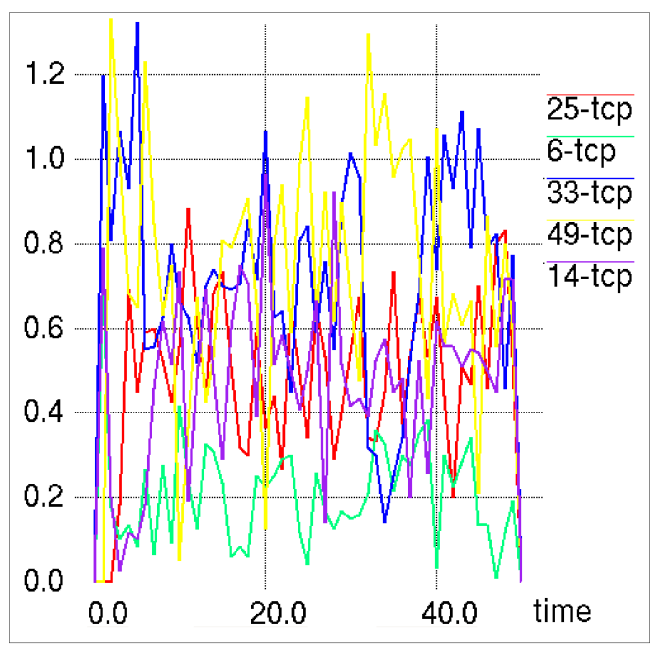

Fig. 11. FTP traffic (tcp packets received by each AP).

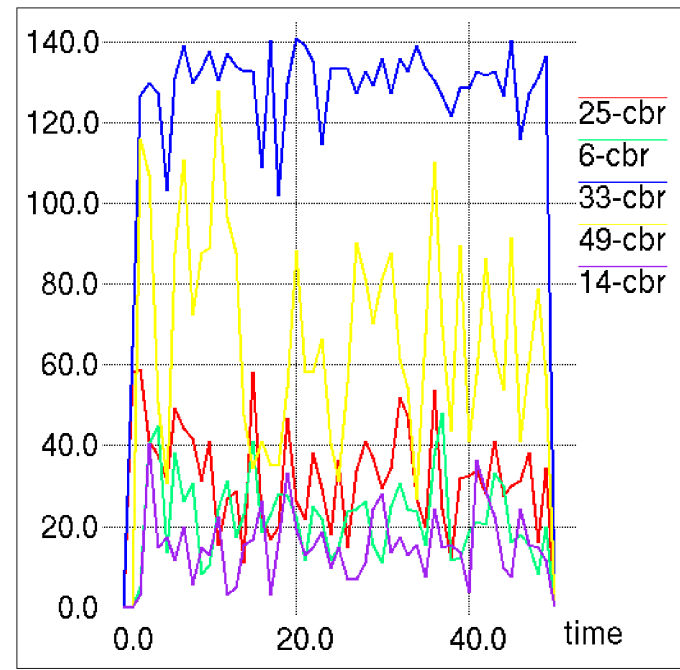

Fig. 12. VoIP traffic (cbr packets received by each AP). switching off RTC/CTS mechanism when the network traffic is expected to be bursty or infrequent, saves power.

The impact of RTS/CTS mechanism should be taken with care. While it is plausible to conclude that channel allocation plays a more significant role than the collision avoidance mechanism, the NS-2 simulation environment has its own limitation. For example, in our simulation, our network model does not support multi-rate transmission. For this reason the RTS/CTS only slightly improves network's performance. It has been pointed out that unlike in single-rate networks, the impact of RTS/CTS in multi-rate networks is profound [15].

\section{REFERENCES}

[1] A. Akella, G. Judd, S. Seshan, and P. Steenkiste. Self-management in chaotic wireless deployment. In The 11th Annual International Conference on Mobile Computing and Networking, 2005.

[2] C.Bettstetter. On the connectivity of wireless multihop networks with homogeneous and inhomogeneous range assignment. In IEEE Vehicular Technology Conference, VTC 2002, pages 1706-1710, 2002.

[3] X. Chao, W. Dargie, and G. Lin. Energy model for h2s monitoring wireless sensor network. In CSE '08: Proceedings of the 2008 11th IEEE International Conference on Computational Science and Engineering, pages 402-409, Washington, DC, USA, 2008. IEEE Computer Society.

[4] Q. Chen, F. Schmidt-Eisenlohr, D. Jiang, M. Torrent-Moreno, L. Delgrossi, and H. Hartenstein. Overhaul of ieee 802.11 modeling and simulation in ns-2. In MSWiM '07: Proceedings of the 10th ACM Symposium on Modeling, analysis, and simulation of wireless and mobile systems, pages 159-168, New York, NY, USA, 2007. ACM.

[5] W. Dargie, C. Xiaojuan, and M. K. Denko. Modelling the energy cost of a fully operational wireless sensor network. Springer Journal of Telecommunication Systems, 2009.

[6] G.Bianchi. Performance analysis of the ieee 802.11 distributed coordination function. IEEE Selected Areas in Communications, 18:535-547, March 2000.

[7] T. Issariyakul and E. Hossain. Introduction to Network Simulator NS2. Springer: Signal \& Communication, 2008.

[8] J. Kephart and D. Chess. The vision of autonomic computing. Computer, 36(1):41-50, 2003.

[9] T.-T. Luong, B.-S. Lee, and C. K. Yeo. Channel allocation for multiple channels multiple interfaces communication in wireless ad hoc networks. In Networking 2008, pages 87-98, 2008.

[10] V. Mhatre, K. Papagiannaki, F. Baccelli, and P. Thomson. Interference mitigation through power control in high density 802.11 wlans. In INFOCOM 2007, 2007.

[11] R. Mochaourab and W. Dargie. A fair and energy-efficient topology control protocol for wireless sensor networks. In CASEMANS '08: Proceedings of the 2nd ACM international conference on Contextawareness for self-managing systems, pages 6-15, New York, NY, USA, 2008. ACM

[12] V. Raman and N. H. Vaidya. Interference aware channel allocation in a multichannel, multi-interface wireless network. In WiNTECH '08: Proceedings of the third ACM international workshop on Wireless network testbeds, experimental evaluation and characterization, pages 111-112, New York, NY, USA, 2008. ACM.

[13] A. research. http://www.abiresearch.com, 2009.

[14] G. Tesauro. Reinforcement learning in autonomic computing. IEEE Internet Computing, 11(1), 2007.

[15] L. Zhang, Y.-J. Cheng, and X. Zhou. Rate avalanche: Effects on the performance of multi-rate 802.11 wireless networks. Simulation Modelling Practice and Theory, 17(3):487-503, 2008.

[16] M. Zimmerling, W. Dargie, and J. M. Reason. Localized poweraware routing in linear wireless sensor networks. In CASEMANS '08: Proceedings of the 2nd ACM international conference on Contextawareness for self-managing systems, pages 24-33, New York, NY, USA, 2008. ACM. 